\title{
Ascites Index - a novel technique to evaluate ascites in ovarian hyperstimulation syndrome: a concept-proof study
}

\author{
Piotr Robert Szkodziak ${ }^{1}$, Piotr Czuczwar ${ }^{1}$, Wojciech Wrona ${ }^{1}$, Tomasz Paszkowski ${ }^{1}$, \\ Filip Szkodziak², Sławomir Woźniak ${ }^{1}$ \\ ${ }^{1} 3^{\text {rd }}$ Chair and Department of Gynecology Medical University in Lublin, Lublin, Poland \\ ${ }^{2}$ Student Research Circle at the $3^{\text {rd }}$ Chair and Department of Gynecology, Medical Univeristy in Lublin, Lublin, Poland
}

\begin{abstract}
Objectives: Controlled ovarian hyperstimulation is an important step in infertility treatment. In some cases, however, ovarian hyperstimulation syndrome (OHSS) can occur. In its severe forms, ascites is likely to develop, associated with dyspnea. The aim of this study was to explore the usefulness of Ascites Index (Asl), a new tool for quantitative determination of ascites in patients with OHSS, to obtain data for planning further trials.

Material and methods: Twelve patients with OHSS and ascites were included in the study. All patients were admitted to the hospital because of abdominal pain and dyspnea due to increasing ascites. Ultrasound measurements of ascites extent were performed in four external quadrants of the abdomen. Pockets of free fluid were measured. The obtained values were totaled, forming the Ascites Index (Asl), similarly to the amniotic fluid index. Because of dyspnea, paracentesis was performed in all cases.

Results: Median Asl at which patients reported dyspnea was $29.0 \mathrm{~cm}$ (range $21.6-38.6 \mathrm{~cm}$ ). At Asl values less than $21.6 \mathrm{~cm}$, no dyspnea was observed in any of the 12 studied patients. To avoid complications, $2000 \mathrm{~mL}$ of ascitic fluid was collected in each patient. After paracentesis, range of Asl decreased to $12.1-14.5 \mathrm{~cm}$.

Conclusions: The proposed Asl seems to be a promising tool for estimating and monitoring the ascites extent in OHSS. It can be estimated using basic ultrasound equipment. Asl requires further studies for standardization and transferability to other causes of ascites.
\end{abstract}

Key words: Ascites Index, ascites, ascites evaluation, ovarian hyperstimulation syndrome

Ginekologia Polska 2018; 89, 4: 182-188

\section{INTRODUCTION}

Ascites is a condition in which an exudate or transudate accumulates in the peritoneal cavity. In normal conditions, the fluid is produced in the peritoneal cavity in a continuous manner. The fluid produced moistens the serosa, facilitating the viscera slide and is subsequently absorbed. In physiological conditions, about $2 / 3$ of fluid from the peritoneal cavity is absorbed into the open lymphatic canals of the diaphragm, reticulum and peritoneum and thanks to negative thoracic pressure is transported to the thoracic spinal canal and left venous angle via the mediastinal trunks. The amount of fluid depends on portal pressure, plasma oncotic pressure, water and sodium retention, quantity of lymph in the body as well as permeability of capillaries and is estimated at about $50 \mathrm{~mL}$. When the production of fluid in the peritoneal cavity is bigger than its absorption, ascites develops [1-3].

Controlled ovarian hyperstimulation is an important procedure used for the treatment of infertility. In some cases, the procedure can be complicated by ovarian hyperstimulation syndrome (OHSS). OHSS manifests as marked enlargement of ovaries, protein-rich fluid accumulation into body cavities and interstitial space ("third space"), which results in condensation of blood concentration and its decreased volume. Accumula- 
tion of protein-rich fluid in the "third space" leads to abdominal distention and increased intra-abdominal pressure. Fully symptomatic OHSS can be complicated by ascites, pericardial exudate, tachycardia with tachypnea, hemorrhage from a ruptured ovary, renal failure, oliguria, hypovolemia, thromboembolic episodes, ischemic stroke, adult acute respiratory distress syndrome, and death. The etiopathogenesis of OHSS has not been fully elucidated. Increased capillary permeability seems essential, caused by substances increasing capillary permeability triggered by human chorionic gonadotropin (hCG), which leads to ascites. In its severe and critical forms, the fluid also occurs in the other body cavities. There are several vasoactive substances: histamine, serotonin, prostaglandins and prolactin. It is currently believed that interleukins are crucial for the development of ascites in OHSS, including the vascular endothelial growth factor (VEGF), tumor necrosis factor- $a$ (TNF- $a$ ) and endothelin-1 [4-9].

The evaluation of ascites extent is necessary for monitoring disease progression and for selecting appropriate treatment methods.

\section{MATERIAL AND METHODS Characteristics of ascites index and its application}

This was a prospective observational concept-proof study verifying the usefulness of Ascites Index (AsI), a new tool for the quantitative determination of ascites in patients with OHSS, compared to the methods commonly used, to obtain data for planning further trials.

The study group consisted of patients after controlled ovarian hyperstimulation diagnosed with the severe form of OHSS accompanied by ascites. The patients were hospitalized in the $3^{\text {rd }}$ Chair and Department of Gynecology,
Teaching Hospital no. 4, Medical University of Lublin in the years 2011-2015 due to OHSS.

Inclusion criteria were enlarged ovary $(>7 \mathrm{~cm}$ in diameter), hematocrit $>45 \%, \mathrm{WBC}>15,000 / \mathrm{mm}^{3}$, oliguria ( $<400 \mathrm{~mL}$ urine per day), creatinine $1.0-1.5 \mathrm{mg} / \mathrm{dL}$, creatinine clearance $>50 \mathrm{~mL} / \mathrm{min}$, estradiol $>5000 \mathrm{pg} / \mathrm{mL}$, severe abdominal pain ( $\geq 6$ point by VAS score), respiratory disorders (dyspnea) and increasing ascites (in physical examination - symmetrical abdominal distension and succussion splash) [7-9]. The exclusion criteria were lack of indications for paracentesis.

Body weight, height and abdominal circumference were measured in all patients. Moreover, the Body Mass Index (BMI) was calculated.

Ultrasound measurements of ascites level were performed through the abdominal wall by one, experienced gynecologist (P.S.) with 23 years of experience in ultrasound in gynecology and obstetrics, using a transabdominal convex ultrasound transducer C3-7IM (frequency range: 3-7 MHz; field of view: 78 degrees) (Medison Accuvix V10/Medison Accuvix V20 Prestige - Seoul, South Korea) and SC1-6 (frequency range: 1-6 MHz; field of view: 60 degrees) (Medison UGEO WS80A Elite - Seoul, South Korea). Examinations were carried out in the dorsal decubitus position without any special preparation of patients. The depth of free fluid pockets was measured perpendicularly to the abdominal circumference tangent (Fig. 1A).

Free fluid areas were found to be located between the diaphragmatic domes, diaphragmatic surface of the right hepatic lobe (upper, right quadrant) and diaphragmatic surface of the spleen (upper, left quadrant) as well as in both iliac fossae between the pelvis minor wall, ascending colon (lower, right quadrant) and descending colon (lower, left quadrant) (Fig. 1B).
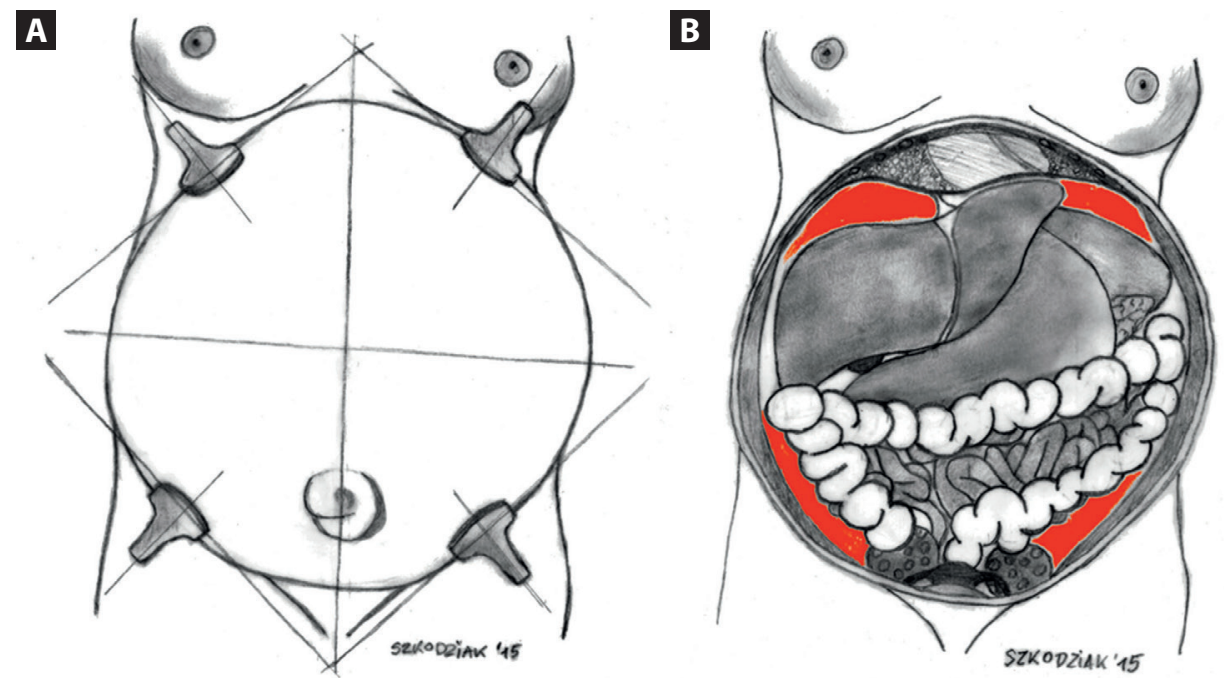

Figure 1. A. Location of an ultrasound probe during Asl examination. B. Anatomical location of ascitic fluid pockets in the peritoneal cavity (orange color) 


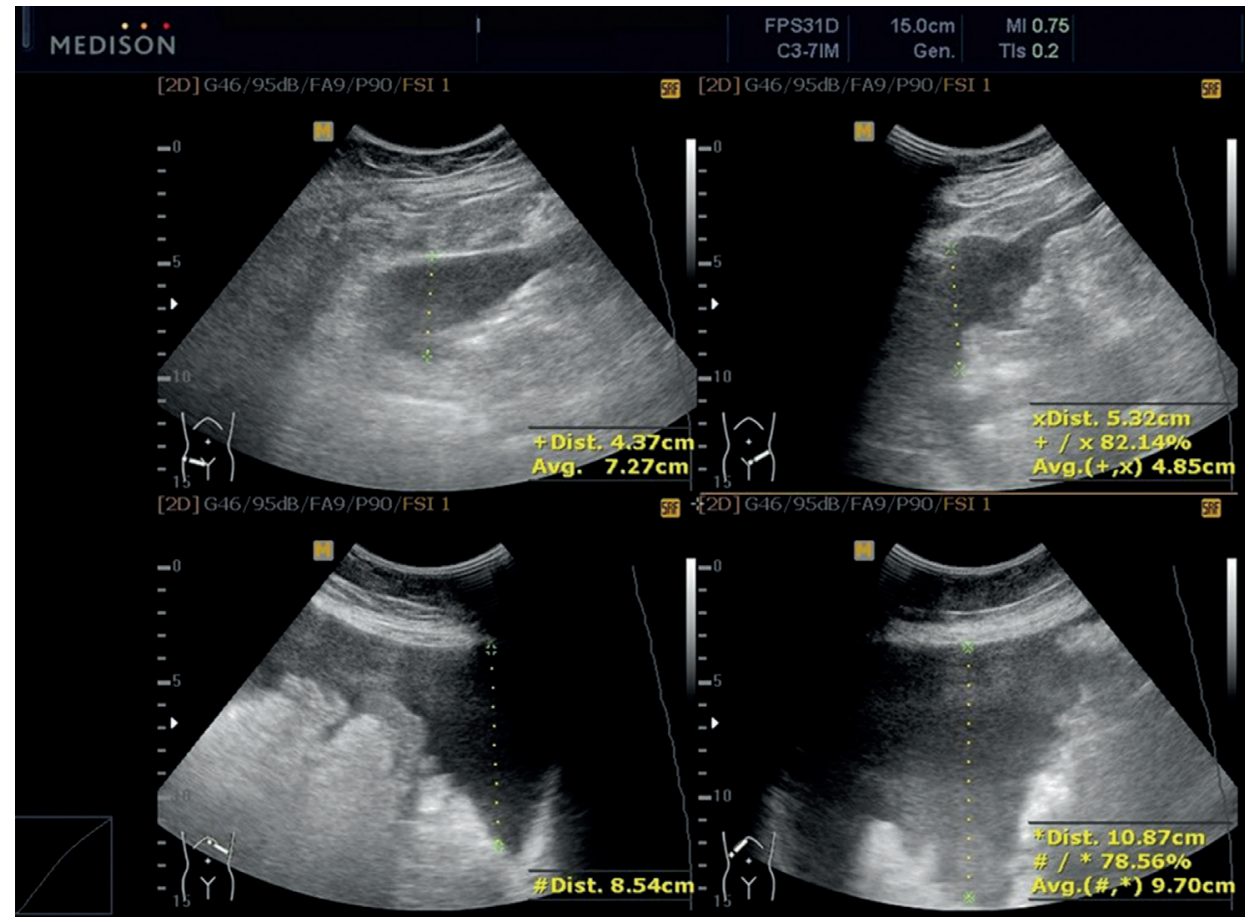

Figure 2. Measurements of the depth of ascitic fluid pockets during ultrasound examination (in this case, Asl was $29.1 \mathrm{~cm}$ )

The values of four measurements were totaled giving the Ascites Index (Asl), similarly to the Amniotic Fluid Index (AFI). The Asl values were presented in centimeters (Fig. 2).

The study design was approved by the Local Bioethics Committee (KE-0254/161/2015) and each patient gave informed consent for participation in the study.

Due to dyspnea, all studied patients underwent paracentesis. To avoid complications associated with excessive protein loss, $2000 \mathrm{~mL}$ of fluid was removed at one time in each patient. Standard treatment was instituted $[3,4,10]$. Using body weight, BMI, abdominal circumference and Asl measurements, the extent of ascites was monitored to evaluate disease progression. Ascites in all patients were monitored until the symptoms of OHSS subsided.

Descriptive data were shown as median (range) due to non-normal distribution. Body weight, BMl, abdominal circumference and Asl of patients before and after paracentesis were compared applying the Wilcoxon signed-rank test. Correlations between Asl and the remaining parameters were tested by the Spearman's Rho test. Statistical analysis was performed using Statistica software (version 10, Statsoft, Tulsa, OK, USA).

\section{RESULTS}

In the analyzed period, 14 patients with OHSS were hospitalized. The study included 12 Caucasian female patients aged 26-34 years. Two patients were excluded from the study. All patients underwent controlled ovarian hyperstimulation prior to an in vitro fertilization programme. In all patients a short agonist protocol to stimulate ovulation was used. Moreover, administering chorionic gonadotropin-alpha was abandoned in all patients due to increased risk of OHSS (serum concentration of estradiol $>5000 \mathrm{pg} / \mathrm{mL}$ ).

Median Asl in patients reporting dyspnea was $29.0 \mathrm{~cm}$ (range 21.6-38.6 cm) and after paracentesis $-12.9 \mathrm{~cm}$ (range 12.1-14.5 cm). At Asl values less than $21.6 \mathrm{~cm}$, no dyspnea was observed in any of the 12 studied patients. Median of Asl percentage decrease after paracentesis was $55.5 \%$ (range 43.5-62.4\%). Median of percentage decrease remaining methods of ascites monitoring (body weight, BMI and abdominal circumference) after paracentesis was less than $5 \%$ in all cases (Tab. 1).

The difference in Asl, body weight, BMI and abdominal circumference before and after paracentesis was statistically significant $(p<0.05)$ (Tab. 1).

There were no statistically significant correlations between Asl and the remaining methods of ascites monitoring before and after paracentesis (Tab. 2).

The detailed results are presented in Table 1 and 2 .

\section{DISCUSSION}

The value of the lower range of Asl at the time of qualification for paracentesis was $21.6 \mathrm{~cm}$. Below this Asl value, we did not observe dyspnea in the study patients and puncture of the peritoneal cavity was not necessary. This applied to two patients who were excluded from the study (Asl was less than $19.0 \mathrm{~cm}$ during hospitalization).

The observed difference Asl pre- and post-paracentesis was statistically significant. Median of percentage decrease 
Table 1. The study parameters in patients with OHSS before and after paracentesis

\begin{tabular}{|c|c|c|c|}
\hline Parameters & Median & Range & $\mathbf{P}$ \\
\hline Pre-procedure body weight (kg) & 60.7 & $53.0-86.5$ & \multirow{2}{*}{$<0.05$} \\
\hline Post-procedure body weight (kg) & 57.9 & $50.3-84.0$ & \\
\hline Post-procedure weight loss (\%) & 4.6 & $2.9-6.6$ & N/A \\
\hline Pre-procedure BMI $\left(\mathrm{kg} / \mathrm{m}^{2}\right)$ & 21.9 & $20.2-31.4$ & \multirow{2}{*}{$<0.05$} \\
\hline Post-procedure BMI (kg/m²) & 20.8 & $18.9-30.5$ & \\
\hline Post-procedure BMI decrease (\%) & 4.6 & $2.9-6.6$ & N/A \\
\hline Pre-procedure abdominal circumference $(\mathrm{cm})$ & 89.4 & $86.1-101.6$ & \multirow{2}{*}{$<0.05$} \\
\hline Post-procedure abdominal circumference $(\mathrm{cm})$ & 86.6 & $82.9-96.2$ & \\
\hline Post-procedure abdominal circumference decrease (\%) & 3.3 & $1.4-5.3$ & N/A \\
\hline Pre-procedure AsI (cm) & 29.0 & $21.6-38.6$ & \multirow{2}{*}{$<0.05$} \\
\hline Post-procedure Asl (cm) & 12.9 & $12.1-14.5$ & \\
\hline Post-procedure AsI decrease (\%) & 55.5 & $43.5-62.4$ & N/A \\
\hline
\end{tabular}

Table 2. Spearman's correlation coefficient (Rho) for Asl and the parameters studied before and after paracentesis

\begin{tabular}{|l|c|c|}
\hline Parameters & Spearman's Rho \\
\hline Asl before paracentesis /body weight before paracentesis & -0.29 \\
\hline Asl after paracentesis/body weight after paracentesis & -0.32 \\
\hline Asl before paracentesis/BMI before paracentesis & 0.24 & NS \\
\hline Asl after paracentesis/BMI after paracentesis & 0.07 & NS \\
\hline Asl before paracentesis/abdominal circumference before paracentesis & -0.26 & NS \\
\hline Asl after paracentesis/abdominal circumference after paracentesis & -0.30 \\
\hline
\end{tabular}

anthropometric methods of ascites monitoring (body weight, BMI and abdominal circumference) after paracentesis was less than $5 \%$. With such a small difference, these methods require very precise measurements. Taking into account the occurrences of observational error, commonly used anthropometric methods may be imprecise [11].

The Spearman correlation between Asl and parallel studied anthropometric parameters (weight, BMI, abdominal circumference) were not statistically significant (Tab. 2). It allows to conclude that in the study group Asl was independent of observed anthropometric parameters.

It is important to note that OHSS may be a life-threatening complication and during controlled ovarian hyperstimulation it is important to minimize the risk of it. However, OHSS is a rare complication and obtaining high numbers in studies investigating this pathology is difficult.

While the mere diagnosis of ascites using the diagnostic methods available is not a problem, quantitative evaluation of the ascites volume is. The peritoneal cavity is roughly spherical, whereas the organs situated in it make its shape irregular. Therefore, calculations of the volume of ascites are a diagnostic problem. The usefulness of quantitative evaluation of ascites seems grounded for assessing treatment outcomes, monitoring fluid increases and earlier planning of decompression procedures [12].

The physical examination is often inaccurate for assessment of ascites and additional diagnostic procedures are required [13]. Non-invasive diagnostic procedures, such as computed tomography or ultrasound examination, are sensitive and specific for ascites diagnosis albeit expensive and currently unable to assess the extent of ascites. The indicator dilution technique (IDT) can be used to calculate the volume of ascites. IDT is performed by quantitative injection of an indicator into the ascitic fluid and subsequent sampling here. The indicator should be a protein bounded tracer or protein bounded dye. After the injection, the abdomen is carefully kneaded and samples of ascitic fluid ate taken 20-60 minutes after indicator injection. The volume of ascites is calculated from the difference in the dilution of the indicator. The method carries the risk of infection, perforation of intestines or hemorrhage $[12,14]$. To date, the methods of monitoring ascites included series measurements of body weight, abdominal circumference and protrusion index, i.e. subjective scoring evaluation of compression-induced deflection of abdominal integuments measured in the line between the xiphoid process and pubic symphysis. Weight changes can 
indirectly indicate decreasing or increasing ascites. Moreover, changes in body weight and structure can be affected by the course of disease inducing ascites and the way of its treatment. Furthermore, abdominal circumference measurements and the protrusion index assume that both the amount of fatty tissue and muscle mass do not change $[15,16]$.

The International Ascites Club (IAC) proposed a classification, in which ascites was divided into uncomplicated and refractory. Uncomplicated ascites is not infected and is unrelated to the development of hepatorenal syndrome. Grade 1 uncomplicated ascites is mild and detectable only on ultrasound; grade 2 is moderate ascites manifesting as slight, symmetrical abdominal distension; grade 3 ascites is evidenced by noticeably marked distension. According to the IAC, refractory ascites is resistant to pharmacological treatment, tends to recur and occurs in about $5-10 \%$ of all ascites cases [17]. The classification by IAC seems of little use in OHSS cases since it is suitable for taking proper therapeutic algorithms rather than treatment monitoring. Moreover, in its severe and critical forms, ascites in the course of OHSS is resistant and hardly ever responses to pharmacological therapy alone.

Ultrasound examinations substantially widen the options of ascites evaluation and enable the localization of free fluid [18].

Ultrasound with a vaginal probe for assessment of the free fluid amount in the peritoneal cavity is commonly used by gynecologists, not only in OHSS cases. Decubitus measurements of pockets of free fluid located in the uterovesical or rectouterine pouch (the Douglas pouch), followed by evaluation of fluid volume based on 3 dimensions (width, depth, height), are precise yet have some limitations. The amount of fluid that can be assessed using this method is small, a range 8-300 mL. In cases of larger fluid amounts, usually present in ascites, the range of penetration of the vaginal probe is too small and the use of transabdominal probe is almost always required [19-21].

Ultrasound evaluation of ascites volume using the transabdominal probe, described by Inadomi et al., is complicated and time-consuming. Patients have to be placed in a horizontal position with the stomach down and support on hands and knees for 10 minutes. In this position, the ascitic fluid collects in the lowest point between the intestinal surface and the posterior surface of the anterior abdominal wall. The examination is performed with a linear probe along the anterior abdominal wall upwards. The largest fluid pocket should be found and measured. Next, the abdominal circumference is measured. It is assumed that the abdominal cavity is modelled as a sphere and the volume is calculated according to the formula: ascitic fluid volume $=1 / 3[\pi \mathrm{d} 2(3 r-d)]$, where ${ }_{\text {, }} \mathrm{d}^{\prime \prime}$ is the width of the largest fluid pocket and "I" is the radius of the abdominal cavity, calculated using the formula ( $r$ = abdominal circumference/2 $\pi$ ) [12].

Moreover, simpler ultrasound methods evaluating ascites extent with the use of a transabdominal probe have been described in literature. Irshad et al. measured the depth of a single free fluid pocket at the site of paracentesis from the abdominal cavity wall to the most superficial intestinal loop before and after the procedure. It has been demonstrated that pre- and post-procedure pocket depths are correlated and can be used for evaluation of ascitic fluid extent in ultrasound examinations [22].

Gerbes et al. proposed a method of evaluating ascites extent, used in gastroenterology, in which the dimension of the Morrison recess is assessed, i.e. the potential peritoneal recess between the lower liver edge and kidney. The examination involves the right epigastrium and is performed in a left lateral decubitus position. The authors have demonstrated the clinical usefulness of the method for monitoring ascites treatment [23].

Based on the method used in the presented study, Alnumeiri et al. suggested the following possible evaluation of ascites volume: after imaging free fluid spaces in the external quadrants of the abdomen, the ascitic fluid volume was estimated using 2 and 3 dimensions (width, depth and height). The sum of the individual measurements provided the total estimated abdominal ascites (TEAA). The method allowed to categorize ascites into 4 grades: I (mild, TEAA $<200-600 \mathrm{~mL}$ ), II (moderate, TEAA > 600-800 mL), III (severe, TEAA > 800$-1000 \mathrm{~mL}$ ), IV (massive, TEAA > 1000-2000 mL). The authors concluded that ascites grade (using ultrasound evaluation of ascitic fluid volume) could reflect the disease etiology [24].

Furthermore, the 5 -grade evaluation of ascites volume was proposed based on computed tomography in patients with neoplastic ascites. Having performed a series of transverse sections of the abdominal cavity, the width of fluid pockets is measured in five places - on both sides in subdiaphragmatic spaces (points $A$ and $B$ ) and paracolonic spaces (points $C$ and $D$ ) as well as in the space between the pubic symphysis and urinary bladder (point E). The subdiaphragmatic and paracolonic measurements are performed in the transverse section while the space in front of the urinary bladder - in the sagittal section. The volume of ascites is calculated according to the following formula: fluid volume $=([A+B+C+D+E] \times 200[\mathrm{~mL}])[25]$.

Computed tomography enables the use a $3 \mathrm{D}$ rendering technique to calculate ascites volume, which provides most reliable data, as compared to other methods described above [25].

The Asl method applies the physical law formulated by Blaise Pascal in the $17^{\text {th }}$ century (known as the Pascal's law). According to this law, the peritoneal cavity is a closed 
spherical reservoir and the fluid inside exerts external pressure, hence the pressure is equal inside the reservoir and it equals the external pressure. The law results from the fact that fluid particles can move in any direction and the pressure put from one side changes the movement of particles in all directions [26]. As a result, free fluid in the peritoneal cavity during pressure equalization passes under the diaphragmatic domes and into both iliac fossae, moving the liver, spleen and intestinal loops medially and forming free fluid pockets (Fig. 1B). The Asl-measured depth of pockets depends on the fluid pressure in the peritoneal cavity whereas the pressure is directly dependent on its amount. Considering the assumptions presented and fixed location of abdominal organs, the rule is - the higher the amount of fluid in the peritoneal cavity, the higher the Asl value.

From the clinical point of view, it is unnecessary to know the volume of fluid in the peritoneal cavity to evaluate the extent of ascites, which has been suggested by some authors mentioned above [12,24, 25]. Even on qualification for paracentesis, the fluid volume is irrelevant as during decompression punctures the peritoneal cavity is not completely emptied, and only the amount of fluid released is limited due to simultaneous loss of albumins. The above-mentioned statement is relevant not only in OHSS but also in other diseases with ascites [3, 4, 27].

Computed tomography is an alternative technique to estimate ascitic fluid volume but lasts longer than the assessment of Asl and requires position changes, what can be inconvenient for ascites patients. Moreover, computed tomography is expensive and contraindicated in pregnancy. In OHSS patients, pregnancy cannot be excluded in all cases.

Ultrasound examinations assessing only the size of one fluid space are equally simple and quick but do not allow to evaluate the distribution of fluid in the peritoneal cavity.

Comparison of pre-and post-paracentesis Asl values and the methods used to date (measurements of body weight, $\mathrm{BMI}$ and abdominal circumference) revealed their statistically significant difference, which suggests the Asl method equally useful for monitoring ascites treatment.

The lack of Asl correlation with body weight, BMI and abdominal circumference allows to anticipate the development of ascites, irrespective of anthropometric characteristics of patients.

Ascites occurs in $15-50 \%$ of patients with neoplastic diseases (ovarian, endometrial, breast, colon, stomach and pancreas cancer). Less common neoplastic causes of ascites include non-Hodgkin lymphoma, prostate cancer, mesothelioma, multiple myeloma and malignant melanoma. Furthermore, ascites can be induced by non-neoplastic diseases, such as portal hypertension, portal vein thrombosis, congestive heart failure, nephrotic syndrome, pancreatitis, tuberculosis and intestinal perforation [28-30]. The diseases mentioned above, as well as their treatment, significantly affect the changes in body weight and structure, which affect both the amount of fatty tissue and also bone and muscle mass [31-33].

Considering the lack of Asl relation to body weight can be useful for monitoring ascites during treatment in cases other than OHSS. However, this thesis should be verified in the group of patients with low and high BMI, because the study did not include a representative group of patients with low and high BMI.

\section{CONCLUSIONS}

To conclude, the methods of monitoring the extent and treatment of ascites involving serial measurements of body weight and abdominal circumference may be imprecise. Therefore, it seems that an accurate and easy implement tool, which could objectively and quantitatively evaluate the extent of ascites using the basic ultrasound equipment, may be useful for evaluating of OHSS course and treatment outcomes.

The proposed Asl seems to be a promising tool for estimating and monitoring the ascites extent in OHSS. It can be estimated using basic ultrasound equipment. Furthermore, this technique may be easily used in outpatient setting.

Asl requires further studies in order to standardization and transferability to other causes of ascites.

\section{Acknowledgments}

None.

\section{Conflict of interest}

None declared.

\section{REFERENCES}

1. Hou W, Sanyal AJ. Ascites: diagnosis and management. Med Clin North Am. 2009; 93(4): 801-817, doi: 10.1016/j.mcna.2009.03.007, indexed in Pubmed: 19577115

2. Saâda E, Follana P, Peyrade F, et al. Pathogenesis and management of refractory malignant ascites. Bull Cancer. 2011; 98(6): 679-687, doi: 10.1684/bdc.2011.1373, indexed in Pubmed: 21636346.

3. Singhal S, Baikati KK, Jabbour Il, et al. Management of refractory ascites. Am J Ther. 2012; 19(2): 121-132, doi: 10.1097/MJT.0b013e3181ff7a8b, indexed in Pubmed: 21192246.

4. Chen $\mathrm{CD}, \mathrm{Wu} \mathrm{MY}$, Chao $\mathrm{KH}$, et al. Update on management of ovarian hyperstimulation syndrome. Taiwan J Obstet Gynecol. 2011; 50(1): 2-10, doi: 10.1016/j.tjog.2011.01.014, indexed in Pubmed: 21482366.

5. Corbett S, Shmorgun D, Claman P, et al. The prevention of ovarian hyperstimulation syndrome. J Obstet Gynaecol Can. 2014; 36(11): 1024-1036, doi: 10.1016/S1701-2163(15)30417-5, indexed in Pubmed: 25574681.

6. Grossman LC, Michalakis KG, Browne H, et al. The pathophysiology of ovarian hyperstimulation syndrome: an unrecognized compartment syndrome. Fertil Steril. 2010; 94(4): 1392-1398, doi: 10.1016/j.fertnstert.2009.07.1662, indexed in Pubmed: 19836016.

7. Nastri CO, Teixeira DM, Moroni RM, et al. Ovarian hyperstimulation syndrome: pathophysiology, staging, prediction and prevention. Ultrasound Obstet Gynecol. 2015;45(4):377-393, doi: 10.1002/uog.14684, indexed in Pubmed: 25302750.

8. Panagiotopoulou N, Byers H, Newman WG, et al. Spontaneous ovarian hyperstimulation syndrome: case report, pathophysiological classification and diagnostic algorithm. Eur J Obstet Gynecol Reprod Biol. 
2013; 169(2): 143-148, doi: 10.1016/j.ejogrb.2013.03.004, indexed in Pubmed: 23561021.

9. Zivi E, Simon A, Laufer N. Ovarian hyperstimulation syndrome: definition, incidence, and classification. Semin Reprod Med. 2010; 28(6): 441-447, doi: 10.1055/s-0030-1265669, indexed in Pubmed: 21082501.

10. Shifman EM, Pogodin OK, Gumeniuk EG, et al. [Intensive care for ovarian hyperstimulation syndrome]. Anesteziol Reanimatol. 2007(4): 77-81, indexed in Pubmed: 17929495

11. Saris W, Revilla M. Correction for Measurement Errors in Survey Research: Necessary and Possible. Social Indicators Research. 2015; 127(3): 1005-1020, doi: 10.1007/s11205-015-1002-x.

12. Inadomi J, Cello JP, Koch J. Ultrasonographic determination of ascitic volume. Hepatology. 1996; 24(3):549-551, doi: 10.1002/hep.510240314, indexed in Pubmed: 8781322

13. Cattau EL, Benjamin SB, Knuff TE, et al. The accuracy of the physical examination in the diagnosis of suspected ascites. JAMA. 1982; 247(8): 1164-1166, indexed in Pubmed: 7057606.

14. Henriksen JH, Møller S. Ascites. Morgan \& Claypool. 2013: 106.

15. Stanley MM, Ochi S, Lee KK, et al. Peritoneovenous shunting as compared with medical treatment in patients with alcoholic cirrhosis and massive ascites. Veterans Administration Cooperative Study on Treatment of Alcoholic Cirrhosis with Ascites. N Engl J Med. 1989; 321(24): 1632-1638, doi: 10.1056/NEJM198912143212403, indexed in Pubmed: 2586565.

16. Wapnick S, Grosberg SJ, Evans MI. Randomized prospective matched pair study comparing peritoneovenous shunt and conventional therapy in massive ascites. Br J Surg. 1979; 66(9): 667-670, indexed in Pubmed: 497660.

17. Moore KP, Wong $F$, Gines $P$, et al. The management of ascites in cirrhosis: report on the consensus conference of the International Ascites Club. Hepatology. 2003; 38(1): 258-266, doi: 10.1053/jhep.2003.50315, indexed in Pubmed: 12830009.

18. Edell SL, Gefter WB. Ultrasonic differentiation of types of ascitic fluid. AJR Am J Roentgenol. 1979; 133(1):111-114, doi: 10.2214/ajr.133.1.111, indexed in Pubmed: 110040.

19. Brezinka C. Tipps und Tricks im Gyn-Ultraschall: Der Abdominalschallkopf beim OHSS. J für Gynäkologische Endokrinol. 2014 Jun 12.; 7(2): 42-43.

20. Khalifé $S$, FalconeT, Hemmings R, et al. Diagnostic accuracy of transvaginal ultrasound in detecting free pelvic fluid. J Reprod Med. 1998; 43(9): 795-798, indexed in Pubmed: 9777619.
21. Von Kuenssberg Jehle D, Stiller G, Wagner D. Sensitivity in detecting free intraperitoneal fluid with the pelvic views of the FAST exam. Am J Emerg Med. 2003; 21(6): 476-478, indexed in Pubmed: 14574655.

22. Irshad A, Ackerman SJ, Anis M, et al. Can the smallest depth of ascitic fluid on sonograms predict the amount of drainable fluid? J Clin UItrasound. 2009; 37(8): 440-444, doi: 10.1002/jcu.20616, indexed in Pubmed: 19618437.

23. Gerbes AL, Gülberg V, Sauerbruch T, et al. German S 3-guideline"ascites, spontaneous bacterial peritonitis, hepatorenal syndrome". Z Gastroenterol. 2011; 49(6): 749-479, doi: 10.1055/s-0031-1273405.

24. Alnumeiri MS, Ayad $\mathrm{CE}$, Ahmed $\mathrm{BH}$, et al. Evaluation of ascites and its etiology using ultrasonography. J Res Dev. 2015; 3(1), doi: 10.4172/jrd.1000119.

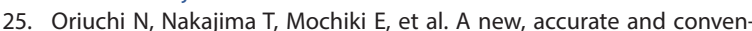
tional five-point method for quantitative evaluation of ascites using plain computed tomography in cancer patients. Jpn J Clin Oncol. 2005; 35(7): 386-390, doi: 10.1093/jjco/hyi109, indexed in Pubmed: 15976067.

26. Fairman JG. Pascal's principle and hydraulics. NASA Official:Tom Benson. 1996. Available from: www.grc.nasa.gov/www/k-12/WindTunnel/Activities/Pascals_principle.html.

27. Cavazzoni E, Bugiantella W, Graziosi L, et al. Malignant ascites: pathophysiology and treatment. Int J Clin Oncol. 2013; 18(1): 1-9, doi: 10.1007/s10147-012-0396-6, indexed in Pubmed: 22460778.

28. Adam RA, Adam YG. Malignant ascites: past, present, and future. J Am Coll Surg. 2004; 198(6): 999-1011, doi: 10.1016/j.jamcollsurg.2004.01.035, indexed in Pubmed: 15194082.

29. Becker G, Galandi D, Blum HE. Malignant ascites: systematic review and guideline for treatment. Eur J Cancer. 2006; 42(5): 589-597, doi: 10.1016/j.ejca.2005.11.018, indexed in Pubmed: 16434188.

30. Gordon FD. Ascites. Clin Liver Dis. 2012; 16(2): 285-299, doi: 10.1016/j. cld.2012.03.004, indexed in Pubmed: 22541699.

31. Aoyagi T, Terracina KP, Raza A, et al. Cancer cachexia, mechanism and treatment. World J Gastrointest Oncol. 2015; 7(4): 17-29, doi: 10.4251/wjgo.v7.i4.17, indexed in Pubmed: 25897346.

32. Magnowski $P$, Wolski $H$, Magnowska $M$, et al. Bone loss in women with malignant genital neoplasms. Ginekol Pol. 2014; 85(12): 955-960, indexed in Pubmed: 25669067.

33. Montano-Loza AJ. Clinical relevance of sarcopenia in patients with cirrhosis. World J Gastroenterol. 2014; 20(25): 8061-8071, doi: 10.3748/wjg. v20.i25.8061, indexed in Pubmed: 25009378. 